\title{
Decontamination of VX with Acid-Activated Clay
}

\author{
Sun Dal Kim ${ }^{*}$ and Yong Han Lee
}

\section{SUPPORTING INFORMATION}

Table S1. Characteristics of metal oxides used in this study.

\begin{tabular}{c|cccccc}
\hline Metal oxides & Product \# ${ }^{\mathrm{a}}$ & $\begin{array}{c}\text { Particle } \\
\text { size }(\mathrm{nm})^{\mathrm{b}}\end{array}$ & $\begin{array}{c}\text { Moisture } \\
\text { content }^{\mathrm{c}} \\
(\mathrm{wt} \%)\end{array}$ & $\begin{array}{c}\text { BET } \\
\text { surface } \\
\text { area } \\
\left(\mathrm{m}^{2} / \mathrm{g}\right)\end{array}$ & $\begin{array}{c}\text { Total pore } \\
\text { volume } \\
\left(\mathrm{cm}^{3} / \mathrm{g}\right)\end{array}$ & $\begin{array}{c}\text { Average } \\
\text { pore } \\
\text { diameter } \\
(\mathrm{nm})\end{array}$ \\
\hline$n \mathrm{Al}_{2} \mathrm{O}_{3}$ & 544833 & $<50$ & 4.0 & 131 & 0.83 & 25.2 \\
$n \mathrm{CaO}$ & 634182 & $<160$ & 1.1 & 33 & 0.07 & 8.3 \\
$n \mathrm{MgO}$ & 549649 & $\leq 50$ & 0.8 & 26 & 0.04 & 6.8 \\
$n \mathrm{TiO}_{2}$ Anatase & 637254 & $<25$ & 3.1 & 96 & 0.25 & 10.2 \\
$n \mathrm{TiO}_{2}$ Mixture & 634662 & $<100$ & 2.9 & 50 & 0.20 & 15.6 \\
$n \mathrm{TiO}_{2}$ Rutile & 637262 & $<100$ & 0.4 & 27 & 0.09 & 13.6 \\
\hline
\end{tabular}

${ }^{a}$ Purchased from Sigma-Aldrich. ${ }^{b}$ Information provided by Sigma-Aldrich. ${ }^{\mathrm{c}}$ Measured by TGA (samples were heated from 20 to $120^{\circ} \mathrm{C}$ at a rate of $20^{\circ} \mathrm{C} / \mathrm{min}$ in a $\mathrm{N}_{2}$ atmosphere, and then the temperature was maintained at $120^{\circ} \mathrm{C}$ for $2 \mathrm{~h}$ ). 
Table S2. Characteristics of metal hydroxides used in this study.

\begin{tabular}{c|cccccc}
\hline $\begin{array}{c}\text { Metal } \\
\text { hydroxides }\end{array}$ & Product \# $\#^{\mathrm{a}}$ & $\begin{array}{c}\text { Particle } \\
\text { size } \\
\left(\mathrm{D}_{50}, \mu \mathrm{m}\right)^{\mathrm{b}}\end{array}$ & $\begin{array}{c}\text { Moisture } \\
\text { content }^{\mathrm{c}} \\
(\mathrm{wt} \%)\end{array}$ & $\begin{array}{c}\text { BET } \\
\text { surface } \\
\text { area } \\
\left(\mathrm{m}^{2} / \mathrm{g}\right)\end{array}$ & $\begin{array}{c}\text { Total pore } \\
\text { volume } \\
\left(\mathrm{cm}^{3} / \mathrm{g}\right)\end{array}$ & $\begin{array}{c}\text { Average } \\
\text { pore } \\
\text { diameter } \\
(\mathrm{nm})\end{array}$ \\
\hline $\mathrm{Al}(\mathrm{OH})_{3}$ & 239186 & 5.32 & 22.2 & 64 & 0.17 & 10.6 \\
$\mathrm{Ca}(\mathrm{OH})_{2}$ & 31219 & 2.83 & 0.6 & 17 & 0.07 & 15.0 \\
$\mathrm{Mg}(\mathrm{OH})_{2}$ & 310093 & 5.63 & 1.1 & 17 & 0.09 & 19.6 \\
$\mathrm{Zr}(\mathrm{OH})_{4}$ & 464171 & 1.69 & 19.6 & 294 & 0.22 & 3.0
\end{tabular}

${ }^{a}$ Purchased from Sigma-Aldrich. ${ }^{b}$ Measured using a laser diffraction analyzer. ${ }^{\mathrm{c}}$ Measured by TGA (samples were heated from 20 to $120{ }^{\circ} \mathrm{C}$ at a rate of $20^{\circ} \mathrm{C} / \mathrm{min}$ in a $\mathrm{N}_{2}$ atmosphere, and then the temperature was maintained at $120^{\circ} \mathrm{C}$ for $2 \mathrm{~h}$ ). 


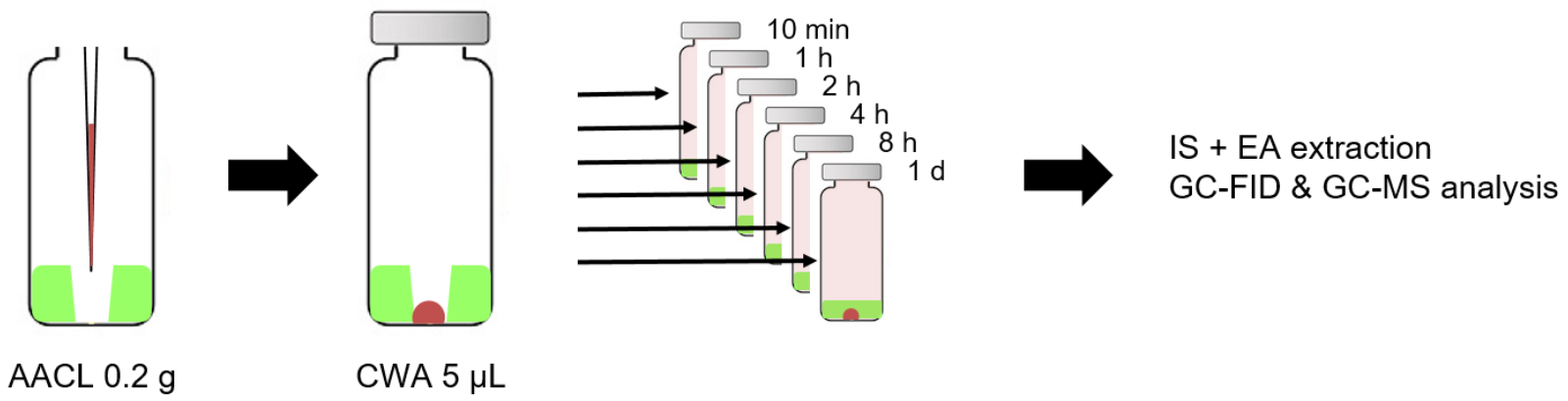

Figure S1. Schematic illustration of the chemical decontamination evaluation procedure. 
Table S3. Ratios of chemically decontaminated CWAs after reaction with metal oxides over different periods of time.

\begin{tabular}{|c|c|c|c|c|c|c|c|}
\hline \multirow{2}{*}{ CWAs } & \multirow{2}{*}{$\begin{array}{c}\text { Contact } \\
\text { time }\end{array}$} & \multicolumn{6}{|c|}{ Chemical decontamination efficiency (\%) } \\
\hline & & $n \mathrm{Al}_{2} \mathrm{O}_{3}$ & $n \mathrm{CaO}$ & $n \mathrm{MgO}$ & $\begin{array}{c}n \mathrm{TiO}_{2} \\
\text { Anatase }\end{array}$ & $\begin{array}{c}n \mathrm{TiO}_{2} \\
\text { Mixture }\end{array}$ & $\begin{array}{l}n \mathrm{TiO}_{2} \\
\text { Rutile }\end{array}$ \\
\hline \multirow{6}{*}{$\mathrm{HD}$} & $10 \mathrm{~min}$ & 2.5 & 0.2 & 0.0 & 0.4 & 0.1 & 0.0 \\
\hline & $1.0 \mathrm{~h}$ & 8.1 & 3.6 & 0.4 & 9.7 & 6.9 & 1.1 \\
\hline & $2.0 \mathrm{~h}$ & 12.8 & 7.1 & 4.0 & 21.1 & 9.6 & 7.4 \\
\hline & $4.0 \mathrm{~h}$ & 29.9 & 11.0 & 7.3 & 26.8 & 12.7 & 13.5 \\
\hline & $8.0 \mathrm{~h}$ & 47.0 & 20.2 & 12.9 & 43.5 & 24.5 & 27.7 \\
\hline & $24 \mathrm{~h}$ & 85.8 & 64.5 & 30.7 & 68.5 & 52.6 & 45.0 \\
\hline \multirow{6}{*}{ GD } & $10 \min$ & 28.3 & 6.6 & 11.1 & 45.5 & 16.5 & 8.0 \\
\hline & $1.0 \mathrm{~h}$ & 55.8 & 27.8 & 19.7 & 72.7 & 34.8 & 33.0 \\
\hline & $2.0 \mathrm{~h}$ & 80.8 & 39.4 & 36.2 & 83.5 & 41.7 & 44.1 \\
\hline & $4.0 \mathrm{~h}$ & 88.9 & 55.0 & 42.2 & 85.3 & 65.5 & 51.5 \\
\hline & $8.0 \mathrm{~h}$ & 93.1 & 56.2 & 50.3 & 91.8 & 77.9 & 90.5 \\
\hline & $24 \mathrm{~h}$ & 97.6 & 83.3 & 77.2 & 99.3 & 97.7 & 94.8 \\
\hline \multirow{6}{*}{ VX } & $10 \mathrm{~min}$ & 1.9 & 0.0 & 1.8 & 95.1 & 84.5 & 9.9 \\
\hline & $1.0 \mathrm{~h}$ & 14.9 & 0.0 & 3.0 & 95.0 & 85.7 & 20.4 \\
\hline & $2.0 \mathrm{~h}$ & 17.2 & 0.0 & 3.1 & 96.0 & 86.4 & 20.8 \\
\hline & $4.0 \mathrm{~h}$ & 18.8 & 0.2 & 4.1 & 97.1 & 89.8 & 21.0 \\
\hline & $8.0 \mathrm{~h}$ & 20.0 & 2.3 & 5.1 & 99.1 & 92.5 & 31.1 \\
\hline & $24 \mathrm{~h}$ & 27.4 & 2.9 & 13.3 & 99.6 & 96.5 & 39.0 \\
\hline
\end{tabular}


Table S4. Ratios of chemically decontaminated CWAs after reaction with metal hydroxides over different periods of time.

\begin{tabular}{|c|c|c|c|c|c|}
\hline \multirow{2}{*}{ CWAs } & \multirow{2}{*}{$\begin{array}{l}\text { Contact } \\
\text { time }\end{array}$} & \multicolumn{4}{|c|}{ Chemical decontamination efficiency (\%) } \\
\hline & & $\mathrm{Al}(\mathrm{OH})_{3}$ & $\mathrm{Ca}(\mathrm{OH})_{2}$ & $\mathrm{Mg}(\mathrm{OH})_{2}$ & $\mathrm{Zr}(\mathrm{OH})_{4}$ \\
\hline \multirow{6}{*}{ HD } & $10 \mathrm{~min}$ & 3.4 & 5.1 & 0.3 & 9.0 \\
\hline & $1.0 \mathrm{~h}$ & 13.9 & 5.8 & 1.6 & 26.3 \\
\hline & $2.0 \mathrm{~h}$ & 19.6 & 7.1 & 3.3 & 47.1 \\
\hline & $4.0 \mathrm{~h}$ & 25.8 & 8.4 & 4.0 & 60.9 \\
\hline & $8.0 \mathrm{~h}$ & 50.2 & 18.0 & 10.2 & 79.1 \\
\hline & $24 \mathrm{~h}$ & 56.2 & 27.3 & 13.9 & 84.4 \\
\hline \multirow{6}{*}{ GD } & $10 \mathrm{~min}$ & 25.8 & 48.4 & 10.9 & 73.3 \\
\hline & $1.0 \mathrm{~h}$ & 52.7 & 80.6 & 20.7 & 93.4 \\
\hline & $2.0 \mathrm{~h}$ & 71.0 & 96.3 & 24.7 & 97.0 \\
\hline & $4.0 \mathrm{~h}$ & 87.4 & 96.2 & 28.3 & 99.3 \\
\hline & $8.0 \mathrm{~h}$ & 90.6 & 98.9 & 40.9 & 98.9 \\
\hline & $24 \mathrm{~h}$ & 99.8 & 99.2 & 71.8 & 100 \\
\hline \multirow{6}{*}{ VX } & $10 \mathrm{~min}$ & 2.6 & 2.5 & 1.7 & 90.4 \\
\hline & $1.0 \mathrm{~h}$ & 13.8 & 11.4 & 2.6 & 92.0 \\
\hline & $2.0 \mathrm{~h}$ & 14.0 & 15.2 & 3.7 & 95.3 \\
\hline & $4.0 \mathrm{~h}$ & 15.5 & 24.4 & 8.9 & 98.9 \\
\hline & $8.0 \mathrm{~h}$ & 18.9 & 34.6 & 11.6 & 98.7 \\
\hline & $24 \mathrm{~h}$ & 32.7 & 79.3 & 14.1 & 99.3 \\
\hline
\end{tabular}




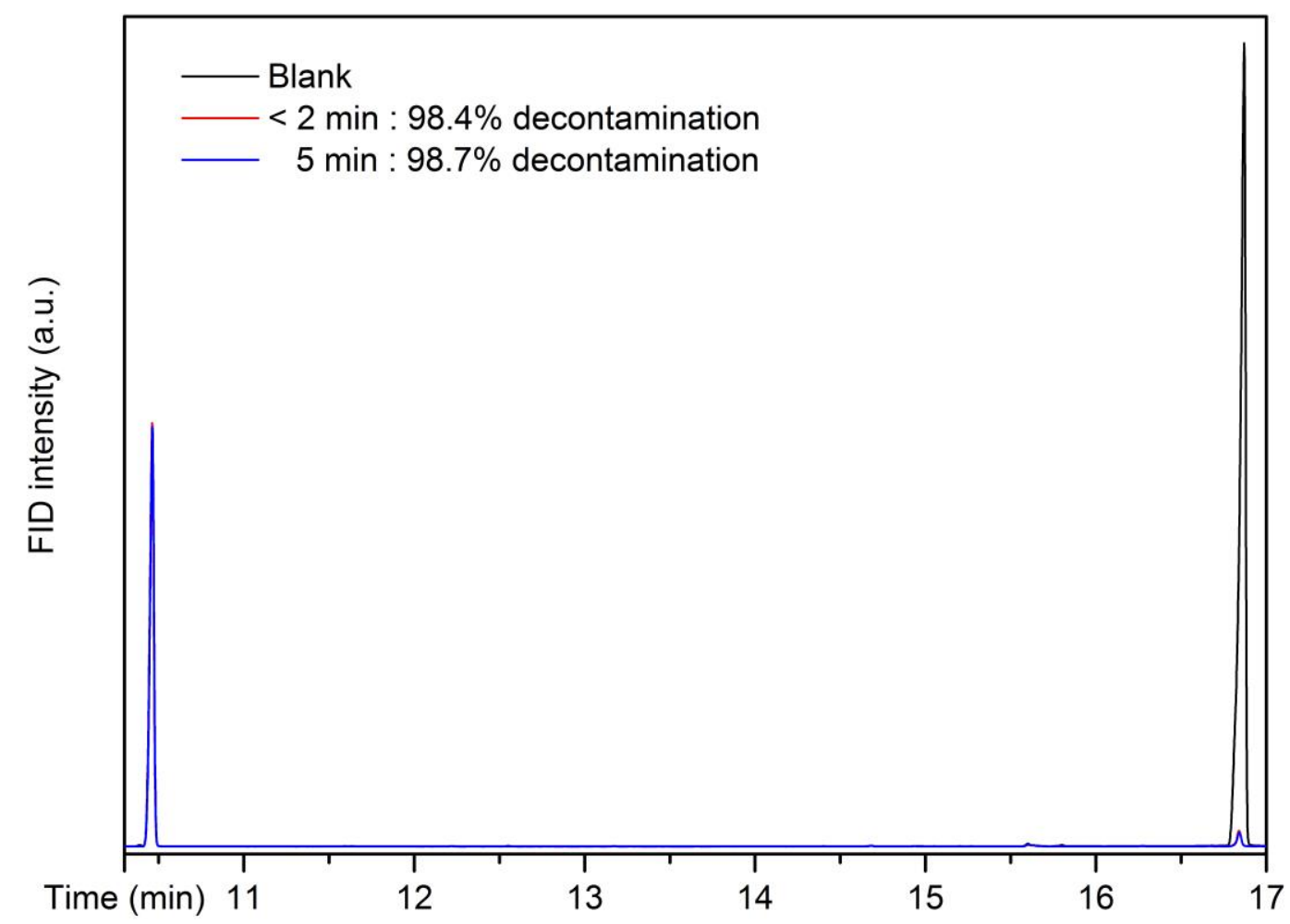

Figure S2. GC-FID chromatograms of samples obtained from the reaction of AACL with VX for $<10$ min. 


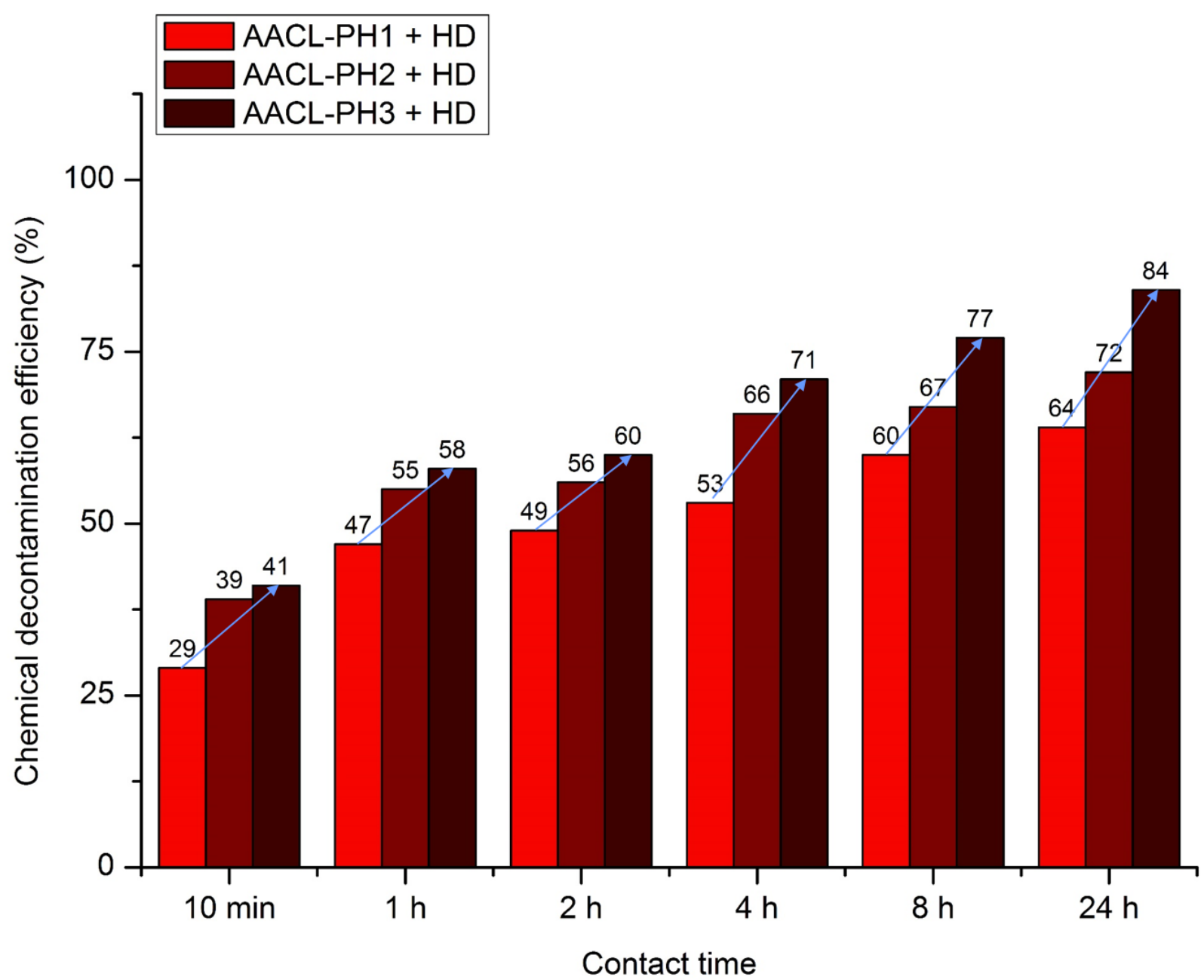

Figure S3. Ratios of chemically decontaminated HD after reaction with AACL-PHs over different periods of time. 
Table S5. Ratios of chemically decontaminated CWAs after reaction with AACL-PHs over different periods of time.

\begin{tabular}{|c|c|c|c|c|}
\hline \multirow{2}{*}{ CWAs } & \multirow{2}{*}{ Contact time } & \multicolumn{3}{|c|}{ Chemical decontamination efficiency (\%) } \\
\hline & & AACL-PH1 & AACL-PH2 & AACL-PH3 \\
\hline \multirow{6}{*}{ HD } & $10 \mathrm{~min}$ & 29.1 & 38.9 & 40.8 \\
\hline & $1.0 \mathrm{~h}$ & 47.0 & 54.9 & 58.3 \\
\hline & $2.0 \mathrm{~h}$ & 48.7 & 56.1 & 59.9 \\
\hline & $4.0 \mathrm{~h}$ & 52.9 & 65.6 & 71.0 \\
\hline & $8.0 \mathrm{~h}$ & 60.3 & 67.4 & 76.9 \\
\hline & $24 \mathrm{~h}$ & 63.6 & 71.9 & 83.8 \\
\hline \multirow{6}{*}{ GD } & $10 \mathrm{~min}$ & 5.3 & 5.7 & 5.3 \\
\hline & $1.0 \mathrm{~h}$ & 7.7 & 7.5 & 7.7 \\
\hline & $2.0 \mathrm{~h}$ & 16.4 & 9.2 & 12.6 \\
\hline & $4.0 \mathrm{~h}$ & 20.9 & 12.8 & 18.5 \\
\hline & $8.0 \mathrm{~h}$ & 26.8 & 14.9 & 22.9 \\
\hline & $24 \mathrm{~h}$ & 31.7 & 17.0 & 25.3 \\
\hline \multirow{6}{*}{ VX } & $10 \mathrm{~min}$ & 96.3 & 83.6 & 68.2 \\
\hline & $1.0 \mathrm{~h}$ & 98.0 & 91.1 & 72.5 \\
\hline & $2.0 \mathrm{~h}$ & 98.0 & 93.3 & 78.1 \\
\hline & $4.0 \mathrm{~h}$ & 98.1 & 95.1 & 84.4 \\
\hline & $8.0 \mathrm{~h}$ & 98.4 & 95.1 & 87.4 \\
\hline & $24 \mathrm{~h}$ & 98.4 & 97.0 & 92.0 \\
\hline
\end{tabular}


Table S6. Characteristics of AACL-PHs.

\begin{tabular}{c|ccccc}
\hline Sample & $\mathrm{pH}^{\mathrm{a}}$ & $\begin{array}{c}\text { Moisture } \\
\text { content }^{\mathrm{b}} \\
(\mathrm{wt} \%)\end{array}$ & $\begin{array}{c}\text { BET surface } \\
\text { area }\left(\mathrm{m}^{2} / \mathrm{g}\right)\end{array}$ & $\begin{array}{c}\text { Total pore } \\
\text { volume } \\
\left(\mathrm{cm}^{3} / \mathrm{g}\right)\end{array}$ & $\begin{array}{c}\text { Average pore } \\
\text { diameter } \\
(\mathrm{nm})\end{array}$ \\
\hline AACL-PH1 & 2.75 & 3.9 & 171 & 0.25 & 5.9 \\
AACL-PH2 & 5.20 & 3.6 & 221 & 0.31 & 5.6 \\
AACL-PH3 & 7.65 & 4.5 & 153 & 0.27 & 7.2 \\
\hline
\end{tabular}

${ }^{\mathrm{a}}$ Determined from measuring a $1 \mathrm{wt} \%$ aqueous solution. ${ }^{\mathrm{b}}$ Measured by TGA (samples were heated from 20 to $120{ }^{\circ} \mathrm{C}$ at a rate of $20{ }^{\circ} \mathrm{C} / \mathrm{min}$ in a $\mathrm{N}_{2}$ atmosphere, and the temperature was maintained for $2 \mathrm{~h}$ ). 
Table S7. Ratios of chemically decontaminated CWAs after reaction with AACL-Ws over different periods of time.

\begin{tabular}{l|c|ccccc}
\hline \multirow{2}{*}{ CWAs } & \multirow{2}{*}{$\begin{array}{c}\text { Contact } \\
\text { time }\end{array}$} & \multicolumn{5}{|c}{ Chemical decontamination efficiency (\%) } \\
\cline { 3 - 7 } & AACL-W1 & AACL-W2 & AACL-W3 & AACL-W4 & AACL-W5 \\
\hline \multirow{7}{*}{ HD } & $10 \mathrm{~min}$ & 42.5 & 17.8 & 9.5 & 4.0 & 2.5 \\
& $1.0 \mathrm{~h}$ & 52.4 & 50.6 & 39.4 & 25.6 & 16.1 \\
& $2.0 \mathrm{~h}$ & 65.9 & 59.5 & 53.6 & 45.8 & 32.9 \\
& $4.0 \mathrm{~h}$ & 67.6 & 61.5 & 63.5 & 54.6 & 42.0 \\
& $8.0 \mathrm{~h}$ & 70.3 & 69.4 & 67.0 & 67.4 & 71.3 \\
& $24 \mathrm{~h}$ & 77.2 & 73.7 & 76.1 & 90.7 & 89.2 \\
\hline \multirow{5}{*}{ GD } & $10 \mathrm{~min}$ & 11.1 & 7.5 & 8.7 & 7.9 & 5.2 \\
& $1.0 \mathrm{~h}$ & 22.9 & 9.9 & 10.8 & 9.7 & 8.9 \\
& $2.0 \mathrm{~h}$ & 24.7 & 12.2 & 13.8 & 12.4 & 13.3 \\
& $4.0 \mathrm{~h}$ & 27.3 & 14.7 & 17.1 & 15.2 & 17.0 \\
& $8.0 \mathrm{~h}$ & 41.4 & 19.1 & 20.2 & 22.8 & 25.1 \\
& $24 \mathrm{~h}$ & 44.7 & 22.9 & 24.7 & 27.9 & 26.8 \\
\hline \multirow{7}{*}{ VX } & $10 \mathrm{~min}$ & 96.9 & 99.2 & 98.3 & 95.9 & 95.2 \\
& $1.0 \mathrm{~h}$ & 97.4 & 99.5 & 98.7 & 97.4 & 97.9 \\
& $2.0 \mathrm{~h}$ & 97.0 & 99.6 & 99.3 & 99.0 & 99.2 \\
& $4.0 \mathrm{~h}$ & 98.2 & 99.7 & 99.7 & 99.7 & 99.8 \\
& $8.0 \mathrm{~h}$ & 98.0 & 99.8 & 99.9 & 99.9 & 100 \\
& $24 \mathrm{~h}$ & 98.2 & 99.9 & 99.9 & 99.9 & 100 \\
\hline
\end{tabular}


Table S8. Ratios of chemically decontaminated CWAs after reaction with ZH-Ws over different periods of time.

\begin{tabular}{|c|c|c|c|c|}
\hline \multirow{2}{*}{ CWAs } & \multirow{2}{*}{ Contact time } & \multicolumn{3}{|c|}{ Chemical decontamination efficiency (\%) } \\
\hline & & ZH-W1 & ZH-W2 & ZH-W3 \\
\hline \multirow{6}{*}{ HD } & $10 \mathrm{~min}$ & 37.4 & 0.0 & 0.0 \\
\hline & $1.0 \mathrm{~h}$ & 56.4 & 0.7 & 0.1 \\
\hline & $2.0 \mathrm{~h}$ & 59.3 & 2.7 & 2.3 \\
\hline & $4.0 \mathrm{~h}$ & 68.2 & 10.5 & 5.0 \\
\hline & $8.0 \mathrm{~h}$ & 79.5 & 18.2 & 15.4 \\
\hline & $24 \mathrm{~h}$ & 83.5 & 30.8 & 24.3 \\
\hline \multirow{6}{*}{ GD } & $10 \mathrm{~min}$ & 48.6 & 65.9 & 81.8 \\
\hline & $1.0 \mathrm{~h}$ & 81.4 & 83.7 & 96.0 \\
\hline & $2.0 \mathrm{~h}$ & 86.2 & 96.3 & 97.5 \\
\hline & $4.0 \mathrm{~h}$ & 86.6 & 96.9 & 99.8 \\
\hline & $8.0 \mathrm{~h}$ & 88.9 & 99.7 & 100 \\
\hline & $24 \mathrm{~h}$ & 92.1 & 100 & 100 \\
\hline \multirow{6}{*}{ vX } & $10 \mathrm{~min}$ & 56.9 & 97.9 & 98.8 \\
\hline & $1.0 \mathrm{~h}$ & 80.1 & 99.9 & 100 \\
\hline & $2.0 \mathrm{~h}$ & 81.2 & 100 & 100 \\
\hline & $4.0 \mathrm{~h}$ & 82.8 & 100 & 100 \\
\hline & $8.0 \mathrm{~h}$ & 92.9 & 100 & 100 \\
\hline & $24 \mathrm{~h}$ & 93.2 & 100 & 100 \\
\hline
\end{tabular}



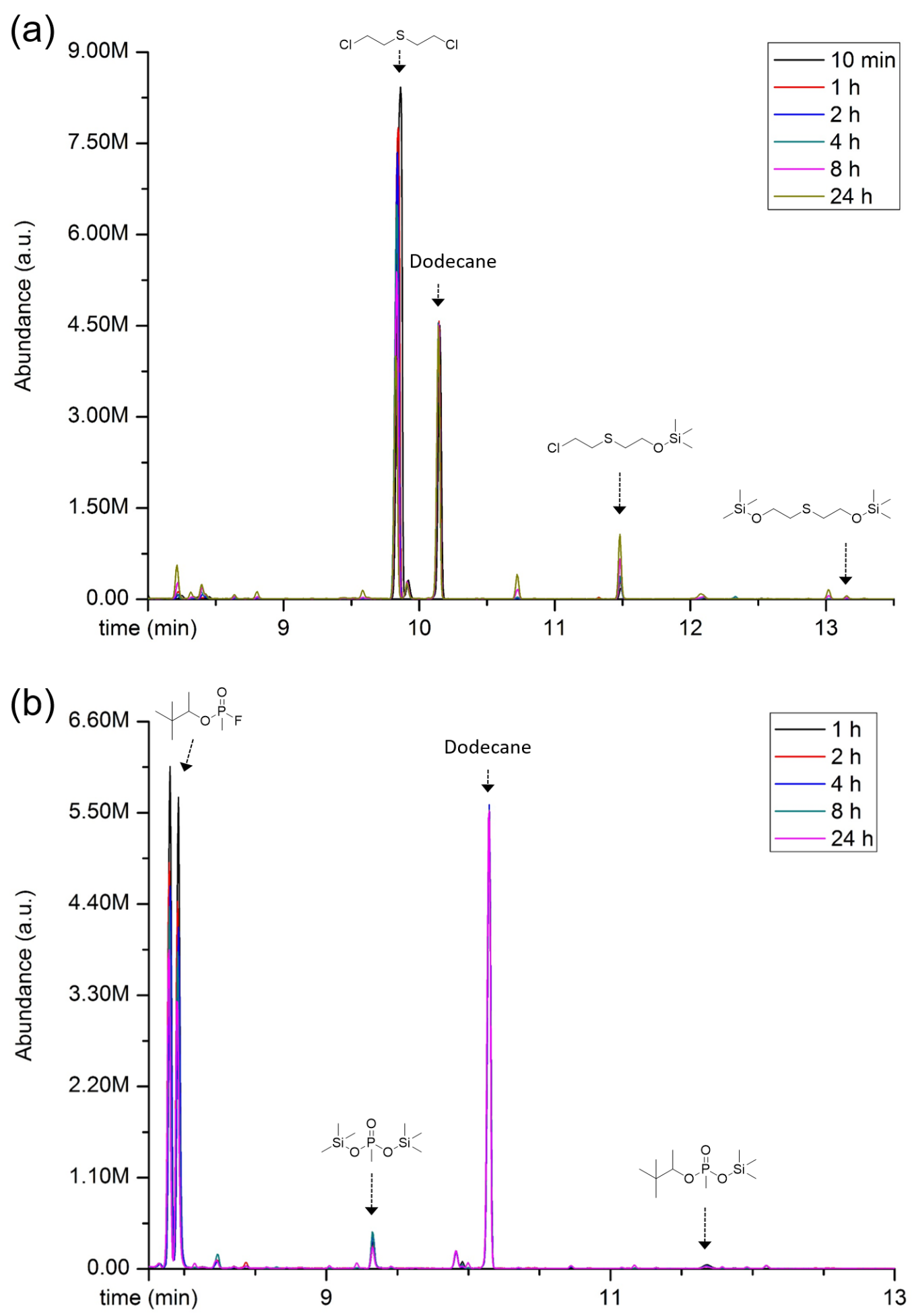

Figure S4. GC-MS total ion chromatograms for samples obtained from the reaction of AACL with (a) HD and (b) GD. 


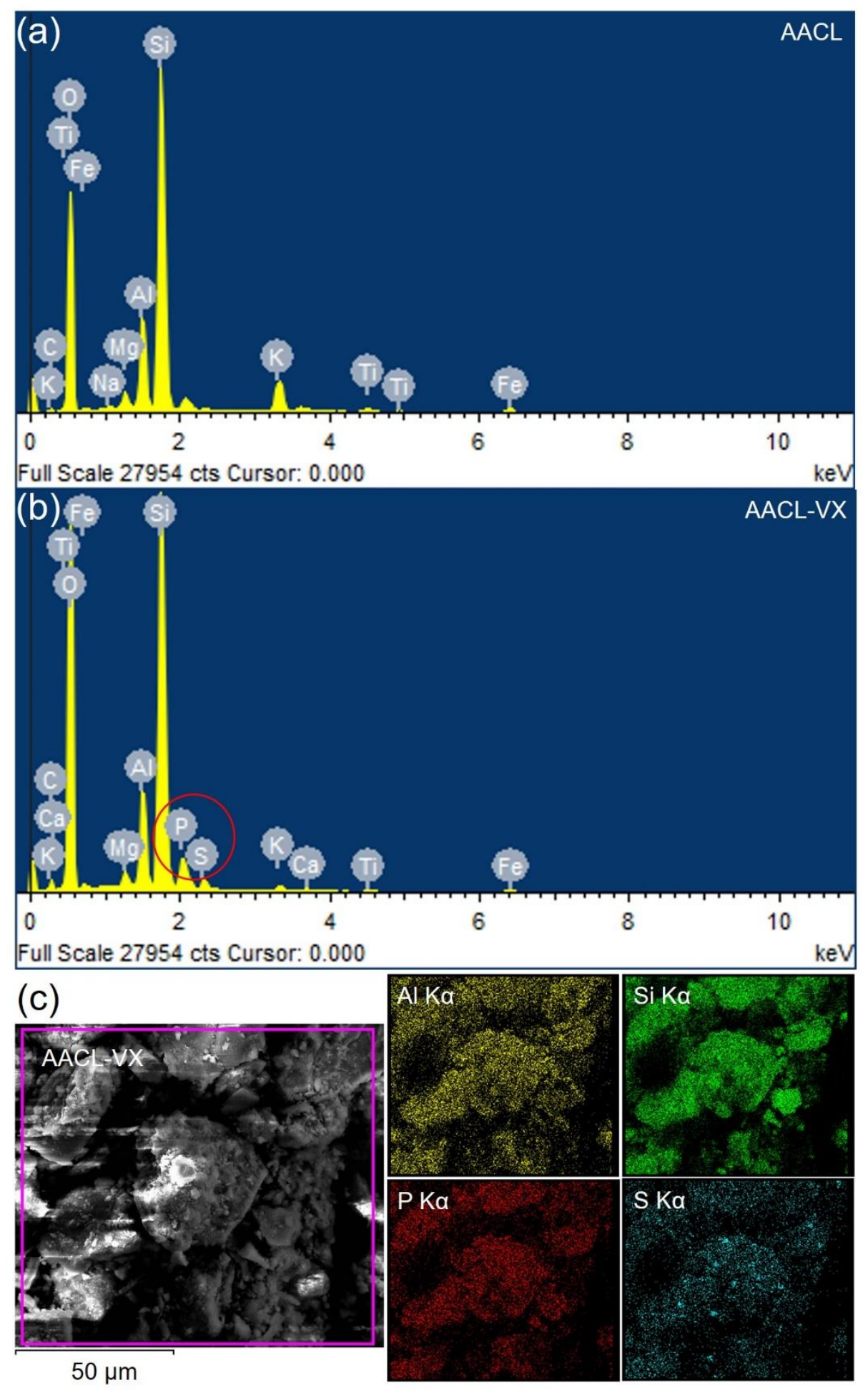

Figure S5. FESEM-EDS spectra of (a) AACL and (b) AACL-VX. (c) FESEM-EDS elemental mapping images of $\mathrm{Al}, \mathrm{Si}, \mathrm{P}$, and $\mathrm{S}$ elements on the AACL-VX surface. 

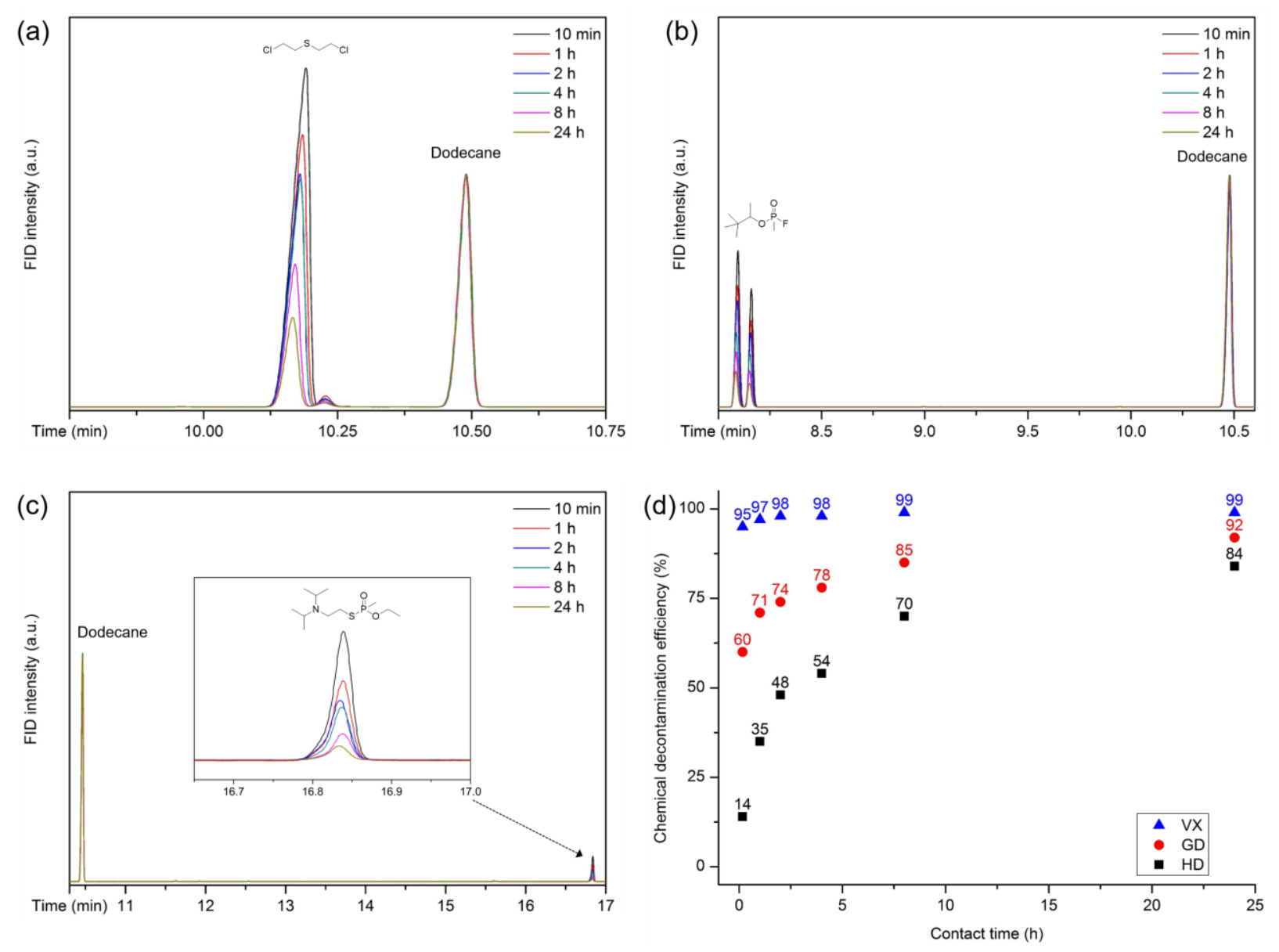

Figure S6. GC-FID chromatograms of samples obtained from the reaction of AACL-ZH with (a) HD, (b) GD, and (c) VX over time. (d) Chemical decontamination efficiencies of CWAs calculated from the corresponding GC-FID chromatograms. 

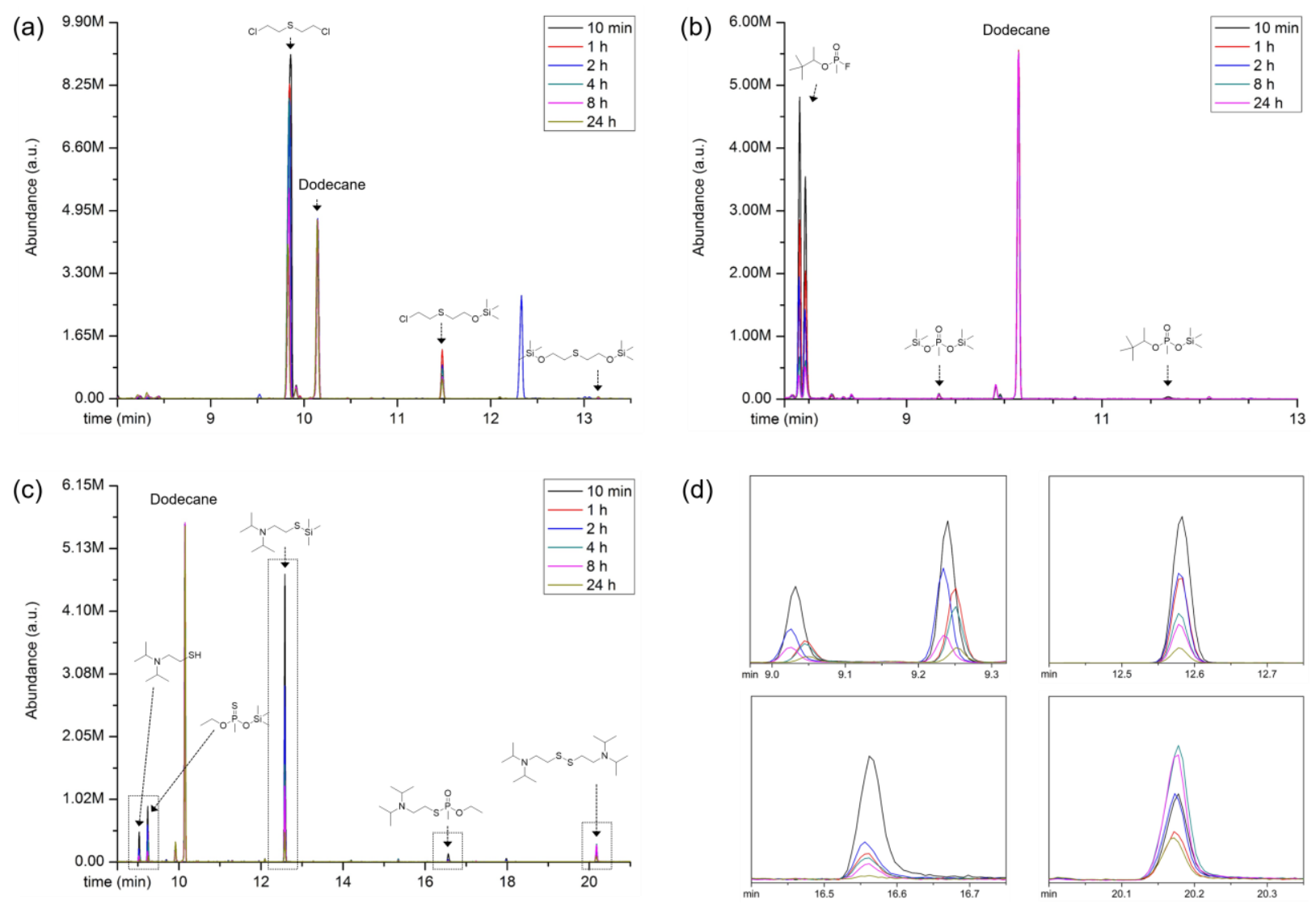

(d)
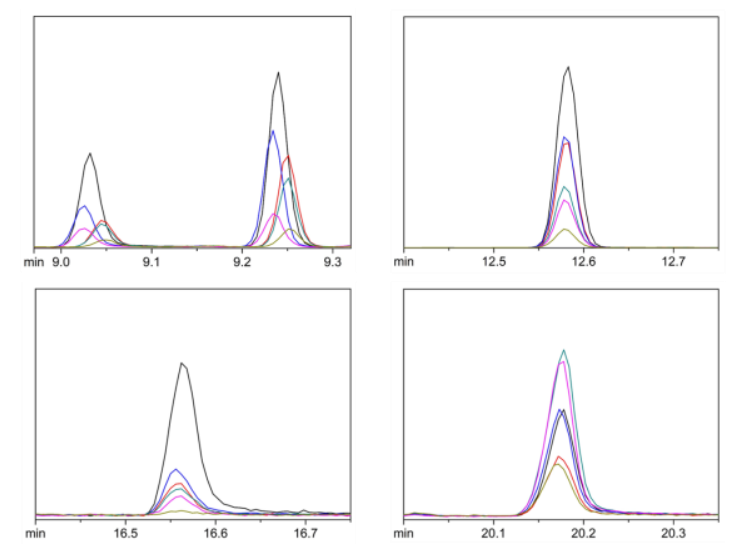

Figure S7. GC-MS total ion chromatograms for samples obtained from the reaction of AACL-ZH with (a) HD, (b) GD, and (c) VX. (d) Enlarged sections of specific ranges identified in (c). 


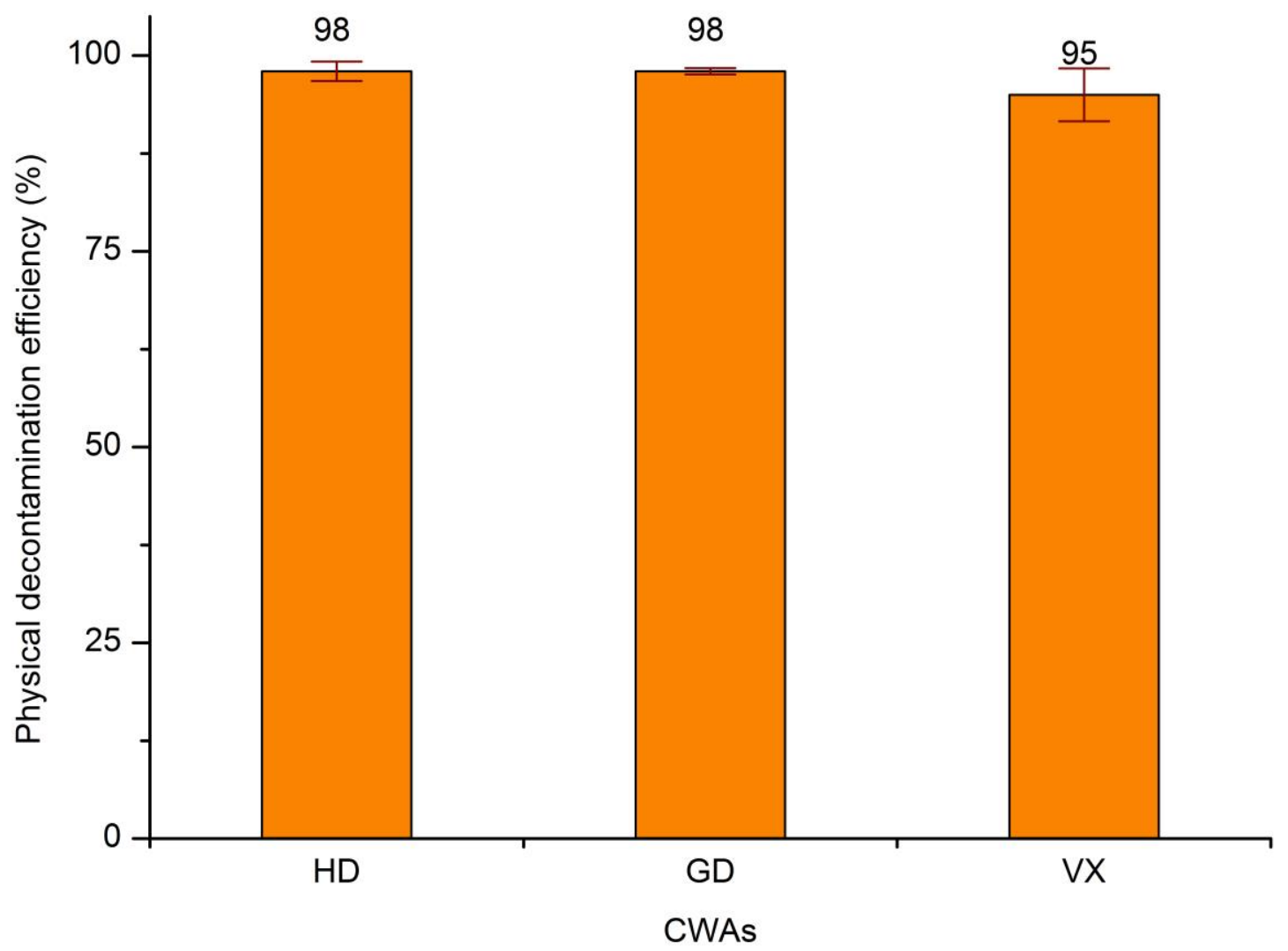

Figure S8. Ratios (as percentages) of CWAs removed from the surface of CARC panels upon adsorption of activated carbon. The BET surface area, total pore volume, and average pore diameter were $1221 \mathrm{~m}^{2} / \mathrm{g}, 0.98 \mathrm{~cm}^{3} / \mathrm{g}$, and $3.2 \mathrm{~nm}$, respectively. The moisture content was 12.2 $\mathrm{wt} \%$. The particle size distributions follow: $\mathrm{D}_{10}=10.59 \mu \mathrm{m}, \mathrm{D}_{50}=36.66 \mu \mathrm{m}$, and $\mathrm{D}_{90}=85.92 \mu \mathrm{m}$. 